\title{
Avaliação por pares na educação médica: um relato das potencialidades e dos desafios na formação profissional
}

\author{
Peer review in medical education: a report of the potentialities and challenges for professional training
}

\author{
Cristina Kaim¹ ${ }^{1}$ | cristinakaim@hotmail.com \\ Bruna Matos de Lima' 1 bbrunamatosl@gmail.com \\ Marcela Araújo de Oliveira Santana' (D) marcela20santana@gmail.com \\ Gustavo Antonio Raimondi' (D) gustavo.raimondi@gmail.com \\ Danilo Borges Paulino' 1 dbpaulino@ufu.br
}

\section{RESUMO}

Introdução: As Diretrizes Curriculares Nacionais (DCN) para o curso de Medicina propõem métodos de ensino e avaliação em que os discentes possam desenvolver criticidade em relação às necessidades de aprendizado. Nesse contexto, a avaliação por pares (AP) apresenta-se como uma estratégia didática capaz de potencializar esse processo. Apesar disso, ainda se observa pouco uso dessa ferramenta na graduação, contudo ela é frequente em congressos científicos e avaliação de periódicos. Diante disso, este relato de experiência busca compartilhar os aprendizados e as reflexões da implementação sistematizada da AP em um componente curricular de Saúde Coletiva do curso de Medicina da Universidade Federal de Uberlândia.

Relato de experiência: O componente em que a atividade foi criada contava com atividades teórico-práticas durante o semestre. Assim, a atividade relatada iniciou-se com a elaboração de pôsteres ao longo do semestre em razão de sua importância em congressos médicos. Esses pôsteres discorriam sobre as vivências dos alunos na atenção primária à saúde. A avaliação desses pôsteres foi feita consecutivas vezes por meio de instrumento de AP pactuado com os estudantes previamente, o qual pontuava critérios estruturais do pôster, bem como seu conteúdo. Os acadêmicos puderam ser avaliados e atuar como avaliadores, sendo supervisionados pelos professores, o que contribuiu para o desenvolvimento da criticidade no processo de aprendizagem.

Discussão: Inicialmente, a atividade gerou estranheza dada a pouca familiaridade dos alunos com a AP, o que foi superado ao longo do semestre. Como limitações, apontamos a necessidade de lidar com as subjetividades no processo avaliativo dos alunos, de formação em feedback e gestão de conflitos, além de possíveis limitações tecnológicas dos discentes.

Conclusão: A AP, embora seja uma estratégia utilizada em outros cenários científicos, possui caráter inovador na graduação e pode promover as competências esperadas para um profissional médico crítico e autônomo, como a familiaridade com a prática do feedback e a capacidade de análise crítica.

Palavras-chave: Educação Médica; Pôster; Revisão por Pares.

\begin{abstract}
Introduction: The Brazilian National Curriculum Guidelines (NCG) for undergraduate courses in medicine propose teaching and assessment methods in which students can develop criticality in relation to learning needs. In this context, Peer Review (PR) represents a didactic strategy capable of enhancing this process. However, there is still little use of this tool in undergraduate training, and frequent use in scientific congresses and evaluation of journals. This experience report seeks to share the learning and reflections of the systematic implementation of the PR in a curricular component of Public Health in a medical undergraduate course at the Federal University of Uberlândia, Brazil.

Experience report: The discipline in which the activity was created had theoretical and practical activities during the semester. Thus, the reported activity started with posters about student experiences in Primary Health Care being made throughout the semester due to its importance in medical congresses. These posters were evaluated consecutively using the PR instrument previously agreed with the students, which scored structural criteria of the poster as well as its content. The students could be evaluated and act as evaluators, being supervised by professors, which contributed to the development of criticality in the learning process.
\end{abstract}

Discussion: Initially, the activity generated strangeness by students because of their lack of familiarity with PR, which was overcome over the discipline. As limitations, we point out the need to deal with subjectivities in the students' evaluation process, training in feedback and conflict management, in addition to possible technological limitations of some students.

Conclusions: $P R$, although a strategy used in other scientific scenarios, has an innovative character in undergraduate courses and can promote the skills expected for a critical and autonomous medical professional, such as familiarity with the practice of feedback and the capacity for critical analysis.

Keywords: Medical Education; Poster; Peer Review.

1 Universidade Federal de Uberlândia, Uberlândia, Minas Gerais, Brasil.

Editora-chefe: Daniela Chiesa

Editor associado: Kristopherson Lustosa Augusto

Recebido em 13/07/20; Aceito em 08/03/21.

Avaliado pelo processo de double blind review. 


\section{INTRODUÇÃO}

Atualmente, a literatura relacionada à educação médica tem apontado que nos cursos de Medicina existe uma cultura conservadora no que tange às avaliações. Assim, tende-se a favorecer o foco no desempenho e no produto, utilizando, quase que exclusivamente, testes padronizados e exames escritos de múltipla escolha, nos quais há pouca interação entre os discentes e destes com os docentes ${ }^{1,2}$.

Ao mesmo tempo, as Diretrizes Curriculares Nacionais (DCN) do curso de graduação em Medicina preveem que os acadêmicos possam aprender de forma ativa, de modo que sejam capazes de identificar e avaliar erros, refletir sobre a própria prática, aprender com autonomia e ter a percepção da necessidade da educação continuada em situações e ambientes protegidos ${ }^{2,3}$, o que estimula a inserção oportuna dos acadêmicos de Medicina na atenção primária à saúde (APS). Essa inserção permite desenvolver competências essenciais para a formação médica, como o trabalho em equipe, o exercício do cuidado em saúde integral, entre outras. Para atingir os objetivos que as DCN propõem, especialmente tendo a necessidade de desenvolver a autonomia dos estudantes ao longo da sua formação e, posteriormente, na atuação profissional, as instituições de ensino são orientadas a utilizar métodos ativos e formativos para as avaliações, como a avaliação por pares (AP)².

Embora pareça uma nova estratégia de avaliação, a AP é utilizada em reuniões científicas, como congressos, e por periódicos desde 1665, promovendo melhora na qualidade, na crítica, na reflexividade, na precisão, no atendimento às normativas éticas e legais, como também na credibilidade do conteúdo a ser publicado 4 .

A AP, durante os processos formativos na e da graduação, baseia-se na aplicação de instrumentos com critérios preestabelecidos para avaliar os resultados ou produtos de aprendizagem de seus pares durante o ensino superior. Essa modalidade de avaliação ativa relaciona-se principalmente com o fornecimento de feedback - informação dada ao estudante que descreve e discute seu desempenho em determinada situação ou atividade, com o objetivo de produzir uma aprendizagem mais abrangente e internalizada, com o uso eficiente do tempo de contato em sala de aula ${ }^{1,2,5,6}$.

Diante disso, observa-se que as etapas da AP são essenciais, já que garantem um feedback consistente e transparente. Essas etapas consistem na descrição da tarefa realizada e em escalas de níveis do desempenho, como "não atende às expectativas", "atende às expectativas", "excede as expectativas" ou simplesmente respostas do tipo "sim" ou "não"1. Além disso, podem ser realizadas de duas formas: sistema aberto, em que a identidade dos autores e revisores é conhecida por ambas as partes; ou sistema duplo-cego, em que a identidade tanto dos autores como dos revisores é desconhecida ${ }^{4}$.

Assim, identifica-se que a AP pode ser utilizada, no ensino superior, para avaliação formativa sobre o desempenho de apresentações orais ou composições escritas, testes de conhecimentos, habilidades ou comportamento esperado ${ }^{1,4}$. Ademais, tem o potencial de aprimorar as competências esperadas para os egressos do curso de Medicina, como a criticidade em relação às necessidades de aprendizado ${ }^{3}$. Isso ocorre devido, por exemplo, à característica da reflexividade da AP na autoanálise e na análise do processo de trabalho com os pares ${ }^{1,2,4}$.

Apesar disso, a AP tem sido utilizada de forma intermitente nos processos de ensino-aprendizagem da graduação em Medicina'. Assim, o objetivo deste relato de experiência é compartilhar os aprendizados e as reflexões de uma experiência sistemática e bem-sucedida de utilização da AP como uma metodologia ativa de ensino-aprendizagem no curso de Medicina da Universidade Federal de Uberlândia.

\section{RELATO DE EXPERIÊNCIA}

Este relato de experiência foi construído pelos docentes idealizadores da experiência e discentes da turma mencionada, enquadrando-se no item VIII do artigo $1^{\circ}$ da Resolução n 510, de 7 de abril de 2016, do Conselho Nacional de Saúde, não necessitando de submissão ao Comitê de Ética em Pesquisa com Seres Humanos ${ }^{7}$.

Durante o componente curricular Saúde Coletiva III, no terceiro período/semestre do curso de Medicina da Universidade Federal de Uberlândia, foi proposta a utilização da AP como estratégia avaliativa dos pôsteres construídos pelos estudantes ao longo do semestre. Esse componente curricular obrigatório tem carga horária teórico-prática de 150 horas, cujo principal objetivo é compreender a determinação social do processo saúde-adoecimento-cuidado, desenvolvendo competências para a atuação em ações de educação em saúde, promoção da saúde e prevenção de agravos. Utiliza metodologias problematizadoras com vivências nas unidades básicas de saúde da família (UBSF) do município. A avaliação nesse semestre é baseada em pôsteres, fichamentos de textos referenciados, projeto de extensão e autoavaliação. Como afirmam as $\mathrm{DCN}^{3}$, o contexto do processo de ensino-aprendizagem explicita algumas necessidades de aprendizagem que, ao serem respondidas, promovem uma aprendizagem significativa. Nesse sentido, os docentes responsáveis pelo módulo consideraram que o contexto de apresentação de pôsteres, como ocorre em eventos científicos, teria um maior potencial de promover um aprendizado significativo em relação à AP.

Segundo Newble et al. ${ }^{8}$, o pôster tem como objetivo atrair a atenção para que determinados conteúdos e/ou 
atividades sejam apresentados e divulgados, fazendo uso de combinações de formas gráficas, cores e informações. Como a forma mais comum de apresentação de trabalhos científicos e pesquisas na área médica é por meio de sessões de pôsteres, é imprescindível que os alunos saibam estruturar e apresentar um pôster, atividades que devem fazer parte das competências adquiridas durante a graduação $0^{8,9}$. O objetivo da utilização da AP nessa atividade era de aprimorar as competências de feedback entre os estudantes, trabalho em equipe, gestão de conflitos e comunicação. Ademais, os docentes responsáveis pelo módulo ponderaram que essa estratégia possibilita a consolidação do conteúdo trabalhado no componente curricular durante a construção, a apresentação e a avaliação do pôster.

Esses estudantes, durante o curso, vivenciam e praticam o feedback utilizando estratégias e instrumentos diferentes e de forma longitudinal e recorrente ao longo do curso, sendo, frequentemente, acompanhados por docentes. Isso ocorre porque o currículo vivenciado por eles se baseia em várias estratégias ativas de aprendizagem, como a aprendizagem baseada em problemas (ABP), estratégia que propicia a construção ativa do seu conhecimento e as habilidades de comunicação, sendo a AP uma ferramenta amplamente utilizada para atingir tais objetivos ${ }^{10,11}$.

Em nossa instituição, o feedback foi apresentado e utilizado desde o primeiro período na ABP. Nos períodos seguintes, a prática de feedback se torna ainda mais presente no currículo, como nas práticas de aprendizagem baseada em equipes ( $\mathrm{ABE}$ ). $A \mathrm{ABE}$ ou team-based learning ( $\mathrm{TBL}$ ) utiliza a $A P$, já que os integrantes das equipes são considerados como aqueles que possuem informações suficientes para avaliação com precisão da contribuição dos pares para a discussão e a construção compartilhada de saberes ${ }^{12}$.

Assim, observa-se que o feedback já era uma prática pedagógica conhecida dos estudantes desde o início do curso e que também tem a possibilidade de estabelecer uma relação entre os diferentes componentes curriculares da formação do discente e sua futura prática profissional. Em relação a essa experiência, o módulo Saúde Coletiva III trabalha integrado ao conteúdo de Método Científico, com o objetivo comum de auxiliar os estudantes na aprendizagem da construção de pôsteres para apresentação em eventos científicos e na escrita de relatos de experiência, com base em vivências de projetos de extensão na APS, envolvendo principalmente ações de promoção da saúde e educação popular em saúde. Esses temas são centrais e trabalhados ao longo do semestre por meio de diferentes metodologias ativas de ensino-aprendizagem sobre as Cartas de Promoção da Saúde, a Política Nacional de Educação Popular em Saúde, entre outros, que serviram como base para as vivências de educação em saúde, desenvolvidas pelos estudantes com a comunidade, em parceria com equipes das UBSF de nosso município.

Durante essas vivências, os acadêmicos acompanharam grupos no formato"sala de espera" com a comunidade, identificando que havia uma relação vertical entre profissionais da saúde e população nessas ações, majoritariamente palestras. A partir dessa observação da realidade e da teorização sobre as temáticas de promoção e educação em saúde, os discentes realizaram com equipes de saúde e comunidade, sob supervisão docente, ações de educação popular em saúde no formato de roda de conversa, acolhendo as demandas da comunidade de forma horizontal e dialógica, e desenvolvendo competências de comunicação, gestão e educação em saúde para a prática médica. Essas vivências, por sua vez, eram as temáticas dos relatos de experiência construídos e apresentados no formato de pôster pelos estudantes.

$\mathrm{Na}$ apresentação do módulo e da programação de aulas durante o início do semestre, os discentes conheceram a proposta da AP e o protótipo do instrumento que seria utilizado para tal. Destaca-se que era a primeira vez que a AP estava sendo explicitada e sistematicamente utilizada no processo de ensino-aprendizagem, em conjunto com o feedback. Ademais, era o primeiro momento do processo formativo em que os discentes participavam ativamente da elaboração e aplicação de instrumentos de avaliação.

Os estudantes puderam analisar a proposta e opinar sobre o formato do instrumento, fazendo sugestões para seu aprimoramento. Após essa discussão, eles concordaram com a atividade proposta e com o instrumento a ser utilizado. No começo do semestre, os discentes construíram um pôster sobre uma vivência realizada na APS que foi avaliado pelos docentes do módulo, que utilizaram o referido instrumento de AP. Essa avaliação foi utilizada para exemplificar quais elementos as avaliações posteriores, a serem realizadas pelos discentes, deveriam/poderiam abranger.

Antes que iniciassem a prática da $\mathrm{AP}$, os discentes estudaram o tema por meio de texto referenciado ${ }^{13}$ e, ao longo do semestre, construíram outros três pôsteres. Dois foram avaliados por uma dupla de estudantes (escolhida por sorteio) por meio do instrumento de AP (Figura 1). A avaliação do último pôster foi realizada por professores convidados, para simularmos uma sessão de apresentação em evento científico.

No terceiro período/semestre do curso, grande parte dos estudantes ainda não tinha vivenciado a exposição de trabalhos em congressos científicos, o que gerou certa curiosidade/medo quando o assunto de apresentação de pôsteres seguida de AP foi mencionado pela primeira vez. Entretanto, ao longo da vivência das avaliações, foi possível entender como o processo se daria, de forma que os estudantes se apropriaram do método proposto, tendo maior fluidez a cada atividade. 
Figura 1. Modelo de instrumento de AP utilizado durante a avaliação dos pôsteres.
Em relação ao pôster $-3,0$ pontos:
* $O$ pôster contém entre seus itens (e estes trazem informações corretas):
1. Cabeçalho com: $(0,25$ ponto)
a) Título do Trabalho (0,05 ponto)
b) Nome dos Autores (0,05 ponto )
c) Nome da Instituição dos(as) estudantes (0,05 ponto) d) Logo da Universidade Federal de Uberlândia $(0,05$ ponto) e) Logo da Secretaria Municipal de Saúde/Prefeitura de Uberlândia ( 0,05 ponto)
2. Introdução. $-0,5$ ponto.
3. Objetivos $-0,5$ ponto.
4. Metodologia $-0,5$ ponto.
5. Descrição da(s) Vivência(s) com:
a) descrição objetiva dos fatos $(\mathbf{0 , 2 5}$ ponto) $\quad$ b) reflexões sobre os fatos $(\mathbf{0 , 2 5}$ ponto) $\quad$ c) fotos/ilustrações $(\mathbf{0 , 2 5}$ ponto)
6. Conclusões/Expectativas. (0,25 ponto).
7. Referências Bibliográficas (de 2 a 3 ) segundo as normas atuais da ABNT. (0,25 ponto)

Feedback - Avaliado pelos docentes responsáveis pelo módulo - 2,0 pontos:

\begin{abstract}
Orientação: Forneçam o feedback com base em aspectos específicos, não de forma geral. Levem em consideração apenas a estruturação do pôster. Impressões subjetivas devem ser expressas como tal. Utilizem-se de linguagem formativa, colaborativa e que não contenha caráter de julgamento ou juízo de valor. Lembrem-se de ressaltar os aspectos positivos e os aspectos que podem ser melhorados e como. Se julgarem necessário, indiquem leituras complementares para aperfeiçoar o processo de trabalho da dupla.
\end{abstract}

Além disso, eles tiveram dificuldades técnicas para confeccionar o pôster, pois, para muitos, essa foi a primeira experiência com essa ferramenta. Para auxiliarem nessa tarefa, os professores apresentaram um tutorial on-line ${ }^{14}$ sobre como construir um pôster e disponibilizaram alguns pôsteres feitos pelos próprios docentes e também por estudantes de semestres anteriores, no intuito de inspirar e tranquilizar os discentes na realização dessa atividade.

As percepções dos alunos sobre a experiência com a AP foram apreendidas em momentos de debate, no formato de roda de conversa, utilizando a estratégia de feedback apreciativo, com a qual eles já estavam familiarizados. Falas positivas foram evidenciadas sobre a possibilidade de vivenciar a "atmosfera de um congresso", realizando e aprimorando a prática para esses momentos. Somado a tal fato, a prática de avaliar o pôster do colega gerou reflexões sobre os próprios pôsteres dos estudantes avaliadores, de maneira que pudessem se inspirar e aprender para construir diferentes pôsteres em atividades futuras, além de aprenderem também com os "erros" dos colegas, produzindo conhecimento e criticidade. Ademais, durante cada apresentação e avaliação, os discentes aprimoraram as competências (conhecimentos, habilidades e atitudes) objetivadas pelo módulo. A evolução dos alunos após o uso do método foi avaliada pela percepção deles durante o feedback, em que refletiram sobre a própria progressão depois da utilização da AP. Além disso, os dois docentes responsáveis pelo componente curricular puderam perceber o crescimento dos estudantes, ao identificarem a incorporação na confecção e apresentação dos pôsteres dos aspectos problematizados na AP, além de observarem como a forma de receber e dar feedback nesse processo se aprimorou, agregando os elementos discutidos nas rodas de conversa.

Com base em Freire ${ }^{15}$, identificamos que os sentimentos que visitaram os estudantes desde a apresentação até a conclusão dessa atividade são esperados e comuns no processo de ensino-aprendizagem baseado na autonomia das pessoas, já que os discentes foram colocados em uma posição ativa perante o seu aprendizado, saindo do modelo tradicional de educação bancária, no qual o professor faz o "depósito" de conteúdos e os alunos são "caixas vazias" que recebem esse conhecimento. Ao auxiliarem os estudantes, os professores responsáveis pelas vivências assumiram o papel de alguém que ajuda o educando a desenvolver o próprio conhecimento, as habilidades e as atitudes, o que possibilitou o desenvolvimento da autonomia ${ }^{15}$. Para Medeiros et al. ${ }^{16}$, essa construção é um compromisso das instituições universitárias para que as pessoas aprendam a aprender por meio do desenvolvimento de competências.

As $\mathrm{DCN}^{3}$ valorizam a formação de um profissional capaz de se comprometer com esse processo, além de estabelecerem a corresponsabilidade do graduando pela própria formação inicial, como o aprender a aprender. Nesse aspecto, as $\mathrm{DCN}^{3}$ destacam a importância das habilidades de comunicação, do trabalho em equipe, do pensamento crítico e de outras características que são colocadas em prática pelo discente 
na realização da $\mathrm{AP}$, assim como durante o feedback da sua avaliação realizada por outros colegas. Além disso, com base nas $\mathrm{DCN}^{3}$, identificamos que a AP pode ser entendida como uma forma de aprendizado em situações e ambientes protegidos e controlados, com a ajuda e orientação dos docentes e o apoio dos colegas ${ }^{3}$, de modo a preparar os discentes para a vivência real de sistematização e divulgação de conhecimentos no ambiente acadêmico. Assim, preocupou-se com a formação médica para além dos aspectos técnicos e assistenciais da profissão.

A prévia pactuação do instrumento solucionou três grandes dificuldades encontradas nas pesquisas sobre a percepção dos discentes: a sistematização dos feedbacks, a associação entre os objetivos e as competências necessárias, e a clareza dos critérios avaliados. Caso não sejam realizados adequadamente, o primeiro ponto gera nos estudantes um sentimento de irrelevância da avaliação recebida; o segundo diminui o engajamento no aperfeiçoamento da aprendizagem, tomando-os inúteis; e o terceiro predispõe um sentimento de incapacidade dos avaliadores ${ }^{2}$.

$\mathrm{Na}$ atividade que aqui relatamos, os avaliadores e avaliados eram identificados, pois estávamos entre pares conhecidos e em processo de aprendizagem, e acreditamos que a identificação facilitaria a comunicação e o aperfeiçoamento da prática. No entanto, os fatores pessoais, subjetivos, em relação às experiências interpessoais anteriores foram identificados como potenciais limitadores para uma avaliação mais objetiva. Houve certa insegurança no que tange à avaliação dos colegas no sentido de os estudantes avaliadores agirem com sinceridade na avaliação e serem mal interpretados pelos discentes avaliados, o que poderia gerar atritos e desavenças para além da atividade.

Em relação a isso, ponderamos que a construção compartilhada do instrumento de AP e a atividade sobre as características de um "bom" feedback são elementos que poderiam minimizar essa questão. Ademais, uma inquietação relatada foi em relação ao apreço demandado por aqueles que avaliavam, de forma que alguns dos estudantes tinham a preocupação de que seus pôsteres poderiam não ser tão bem julgados quanto os que eles avaliaram, o que poderia gerar um sentimento de "injustiça".

Um desafio para a realização da AP é limitar os fatores pessoais e subjetivos dentro da avaliação, já que o conceito avaliativo atribuído pelos avaliadores pode ser influenciado mais pelas impressões pessoais que eles têm do avaliado do que pelo desempenho deste, o que é denominado "efeito halo". Uma solução encontrada por Domingues et al. ${ }^{13}$ foi a confidencialização dos avaliadores. Isso pode ser feito por meio do método duplo-cego, no qual o avaliador desconhece a identidade daquele que avalia, sendo o contrário verdadeiro, ou seja, o avaliado não sabe quem o avaliou.

Além disso, aprendemos com essa experiência que seria importante para os estudantes uma formação prévia em gestão de conflitos; referimo-nos aqui aos processos autoorganizados, em que os envolvidos podem reconstruir relações com os outros e consigo mesmos ${ }^{17}$. Os métodos de gestão de conflitos possuem diversos princípios, como atender às necessidades das partes interessadas, promover estratégias para satisfazer as expectativas e minimizar o conflito afetivo ${ }^{17,18}$. Existem diversas estratégias nesses métodos, entre as quais se destaca a mediação, na qual se utiliza-se de uma terceira parte, chamada de mediador ${ }^{19}$. Isso pode ser integrado à vivência de $A P$, já que o docente responsável pode mediar os conflitos gerados pelos feedbacks ${ }^{18}$ ou até mesmo um terceiro colega poderia atuar e aprimorar-se na prática de gerenciar e mediar conflitos, algo que com certeza lhe será útil no trabalho em equipes multiprofissionais de saúde, no presente e no futuro.

\section{CONCLUSÕES}

O uso da AP como metodologia ativa de ensinoaprendizagem proporciona aos estudantes a possibilidade de aprimorar habilidades necessárias para a prática médica que estão previstas nas $\mathrm{DCN}^{3}$, como trabalho em equipe, feedback, tomada de decisões e gestão de conflitos. Além disso, foi potencializado o desenvolvimento da autonomia dos discentes ao assumirem uma posição ativa na construção de conhecimentos, inclusive do próprio instrumento de AP. Nesse sentido, também destacamos a importância de uma relação educando-educador mais horizontalizada para potencializar os processos de ensino-aprendizagem ativos e que tem como uma de suas premissas a autonomia.

Como limitações, apontamos a necessidade de lidar com as subjetividades no processo avaliativo e de formação em feedback e gestão de conflitos, e a demanda por uma postura de facilitador do processo de ensino-aprendizagem. Por parte dos discentes, o trabalho com a ferramenta do pôster exige recursos tecnológicos que podem não estar acessíveis a todos. Deve-se ter atenção para, nas possibilidades de cada instituição, permitir que todos tenham acesso à formação necessária para o uso da AP, bem como aos recursos humanos e tecnológicos fundamentais para essa prática.

Quando da execução dessa atividade em outros contextos, recomendamos o seguinte: 1 . deve-se realizar uma formação prévia em gestão de conflitos e feedback; 2. o instrumento utilizado com os critérios de avaliação deve ser partilhado com os discentes antes do início das atividades, de modo a permitir que as sugestões deles possam aprimorá-lo; e 3. devese partilhar com os discentes a decisão de utilização ou não do método duplo-cego. Essas recomendações têm como objetivo, 
principalmente, minimizar os conflitos durante a execução dos feedbacks, potencializando o desenvolvimento de competências que a AP pode proporcionar na formação médica.

\section{AGRADECIMENTOS}

À direção da Faculdade de Medicina, à coordenação do curso de Medicina e ao Departamento de Saúde Coletiva (DESCO) da Faculdade de Medicina (FAMED) da Universidade Federal de Uberlândia (UFU).

\section{CONTRIBUIÇÃO DOS AUTORES}

Danilo Borges Paulino e Gustavo Antonio Raimondi, além de serem responsáveis pela idealização e confecção do projeto que originou este relato de experiência, participaram ativamente das demais etapas de elaboração deste manuscrito. Todos os autores participaram da execução do projeto e da redação e revisão do manuscrito.

\section{CONFLITO DE INTERESSES}

Os autores declaram não haver conflito de interesses neste estudo.

\section{FINANCIAMENTO}

Declaramos que não houve financiamento para a realização desta pesquisa.

\section{REFERÊNCIAS}

1. Eldredge JD, Bear DG, Wayne SJ, Perea PP. Student peer assessment in evidence-based medicine (EBM) searching skills training: an experiment. J Med Libr Assoc. 2013;101(4):244-51.

2. Costa CB. Autoavaliação e avaliação pelos pares: uma análise de pesquisas internacionais recentes. Revista Diálogo Educacional. 2017;17(52):431-53.

3. Brasil. Resolução no 3, de 20 de junho de 2014. Institui Diretrizes Curriculares Nacionais do Curso de Graduação em Medicina e dá outras providências. Diário Oficial da União; 23 jun 2014. Seção 1.
4. Jenal S, Vituri DW, Ezaías GM, Silva LA, Caliri MHL. O processo de revisão por pares: uma revisão integrativa de literatura. Acta Paul Enferm. 2012;25(5):802-8.

5. Allibaih $M$, Khan LM. Weaving together peer assessment, audios and medical vignettes in teaching medical terms. Int J Med Educ. 2015;6(6):172-8.

6. Zeferino $\mathrm{AB}$, Domingues $\mathrm{RCL}$, Amaral $\mathrm{E}$. Feedback como estratégia de aprendizado no ensino médico. Rev Bras Educ Med. 2007;31(2):176-9.

7. Brasil. Resolução no 510, de 7 de abril de 2016. Dispõe sobre as normas aplicáveis a pesquisas em Ciências Humanas e Sociais. Diário Oficial da União; 24 maio 2016. Seção 1.

8. Newble D, Cannon R. A handbook for medical teachers. 4th ed. Boston: Kluwer Academic; 2001. p. 56-69.

9. Lorenzoni PJ, Souza RCA, Kohara SK, França JCB, Rodrigues GA, Carvalho JGR. O pôster em encontros científicos. Rev Bras Educ Med. 2007;31(3):304-9.

10. Barrows HS. Problem-based learning in medicine and beyond: a brief overview. In: Wilkerson L, Gijselaers WH, editors. Bringing problem-based learning to higher education: theory and practice. San Francisco: JosseyBass; 1996. p. 3-12.

11. Barrows HS, Tamblyn RM. Problem-based learning: an approach to medical education. New York: Springer; 1980.

12. Bollela VB, Senger MH, Tourinho FSV, Amaral E. Aprendizagem baseada em equipes: da teoria à prática. Medicina (Ribeirão Preto). 2014;47(3):293-300.

13. Domingues RCL, Amaral E, Zeferino AMB. Auto-avaliação e avaliação por pares: estratégias para o desenvolvimento profissional do médico. Rev Bras Educ Med. 2007;31(2):173-5.

14. Poster Session Online. How to build a poster [acesso em 4 maio 2020] Disponível em: http://www.postersessiononline.eu/diseno_powerpoint.asp.

15. Freire P. Pedagogia da autonomia. São Paulo: Paz e Terra; 1996.

16. Medeiros, RM, Stédile NLR, Calus SM. Construção de competências em enfermagem. Caxias do Sul: Educs; 2001.

17. Claro RFS, Cunha PFSS. Estratégias de gestão construtiva de conflitos: uma perspetiva dos profissionais de saúde. Psicol Saúde Doenças. 2017;18(1):65-8.

18. Cunha $P$, Meneses $R$, Oliveira MC. Gestão de conflitos na área da saúde: uma proposta de reflexão. Arq Med. 2013;27(3):132-4.

19. Campos L. Mediação de conflitos: enquadramentos institucionais e posturas epistemológicas. In: Vasconcelos-Sousa J, coordenador. Mediação em acção. Coimbra: MinervaCoimbra; 2008. p. 167-95. 